\title{
LIPOMAS OF THE QUADRIGEMINAL PLATE: A REPORT OF TWO CASES
}

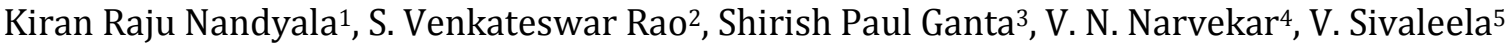

\section{HOW TO CITE THIS ARTICLE:}

Kiran Raju Nandyala, S. Venkateswar Rao, Shirish Paul Ganta, V. N. Narvekar, V. Sivaleela. "Lipomas of the Quadrigeminal Plate: A Report of Two Cases". Journal of Evolution of Medical and Dental Sciences 2015; Vol. 4, Issue 24, March 23; Page: 4229-4231, DOI: 10.14260/jemds/2015/610

ABSTRACT: Lipomas are benign tumors which can rarely involve the central nervous system (CNS), the incidence not exceeding $0.5 \%$ of all the intracranial tumors. ${ }^{1,2}$ The intracranial lipomas are usually small, well circumscribed and have any age or sex predominance except for the spinal lipomas which predominate in children and young adults. They are located more frequently in the corpus callosum, tuber cinereum, infundibulum, quadrigeminal plate and cisterna ambiens. In the last location the lesion may lie on the midbrain tectum and the superior vermis. In the present paper we report two cases of left quadrigeminal plate lipomas who presented with headache and seizures, both of which could be managed by medical treatment.

KEYWORDS: Corpus callosum Lipoma, Quadrigeminal plate lipoma, Intracranial lipoma.

\section{CASE REPORTS:}

CASE I: A 30-year-old man presented with headache of 1-year duration with on and off seizures of 1 month duration. A cranial computed tomography (CT) revealed a normal cranial vault as well as cerebral and cerebellar hemispheres. A CT scan showed a hypodense lesion (-48 Hounsfield units) with arc like calcification in left half of quadrigeminal plate cistern. (Fig. 1)

Magnetic resonance imaging (MRI) brain showed hyperintense lesion on T1-weighted images and relatively high intensity on T2-weighted images. The findings were consistent with fat and a diagnosis of quadrigeminal plate cistern lipoma was made. The patient was managed conservatively and the patient's headaches subsided and seizures were under control with conservative management.

Case II: This 19 year old girl had head ache for 4 weeks before admission. There was no neurological deficit. A CT scan showed low-density lesion of the left quadrigeminal plate cistern. (-80H.U). ${ }^{3}$

Axial and coronal MR scans were done. Magnetic resonance imaging (MRI) brain showed hyperintense lesion on T1-weighted images (Fig. 3) and relatively high intensity on T2-weighted images and hypointense lesion on FLAIR image. The findings were consistent with fat and a diagnosis of quadrigeminal plate cistern lipoma was made.4,5

Patient was treated conservatively on medical treatment. No surgery was performed. ${ }^{4}$

DISCUSSION: An intracranial lipomas are not a tumours as such, but rather a result of "abnormal differentiation of embryologic meninx primitiva. They are frequently associated with abnormal development of adjacent structures, supporting the so called "brain dysraphism" theory.

CLINICAL PRESENTATION: They are usually asymptomatic, and either found incidentally or as a result of investigation of related malformations and their presentation (e.g. headache, mental retardation, epilepsy etc.). 


\section{LOCATION:}

- Peri-callosal lipoma: $45 \%$, associated with agenesis of the corpus callosum in approximately $50 \%$ of cases. ${ }^{1}$

- Quadrigeminal cistern lipoma: 25\%, associated with underdevelopment of the inferior colliculus.

- Suprasellar cistern lipoma: $15 \%$.

- Cerebellopontine angle lipoma: 10\% (the facial nerve and vestibulocochlear nerve often courses through the lipoma - see case 1).

- Sylvian fissure: $5 \%$.

RADIOGRAPHIC FEATURES: The characteristic finding on both CT and MRI is of a mass which has appearances consistent with fat. CT (negative HU values) and MRI with and without fat saturation are able to make the diagnosis easily. ${ }^{5}$

Often the lipomas are traversed by cranial nerves and adjacent vessels.

Some peripheral calcification may be present

DIFFERENTIAL DIAGNOSES: The differential is essentialy that of masses which contain fat, and therefore includes:

- Intracranial dermoid if ruptured will often have multiple droplets scattered through the subarachnoid space. Usually midline.

- Intracranial teratoma

- lipomatous transformation of neoplasm: PNET, Ependymoma, Glioma.

On MRI, if no fat saturated sequences are available then a number of other possibilities should be entertained, which also have high T1 signal. ${ }^{5}$

- Thrombosed Berry aneurysm: often will have calcified rim, and haemosiderin staining on gradient echo/ SWI sequences.

- White dermoid: rare, and will restrict on DWI.

\section{REFERENCES:}

1. Krainer L. Die Hirn - und Ruckenmarkslipome. Virchow Arch [A]. 1935; 295: 107-142.

2. Donati F, Vassella F, Kaiser G, Blumberg A. Intracranial lipomas. Neuropediatrics. 1992; 23: 3238.

3. Truwit CL, Barkovich AJ. Pathogenesis of intracranial lipoma: an MR study in 42 patients. AJNR. 1990; 11: 665-674.

4. Verga P. Lipoma ed osteolipomi della pia madre. Tumori. 1929; 15: 321-357.

5. Maiuri F, Cirillo S, Simonetti L, De Simone MR, Gangemi M. Intracranial lipomas: diagnostic and therapeutics considerations. J. Neurosurg. Sci. 1988; 32: 161-167. 


\section{CASE REPORT}

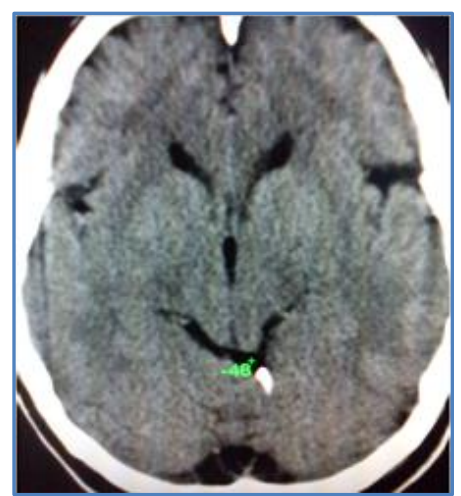

Fig. 1: CT scan showed fat-density lesion of the left quadrigeminal plate cistern with arc shaped calcification

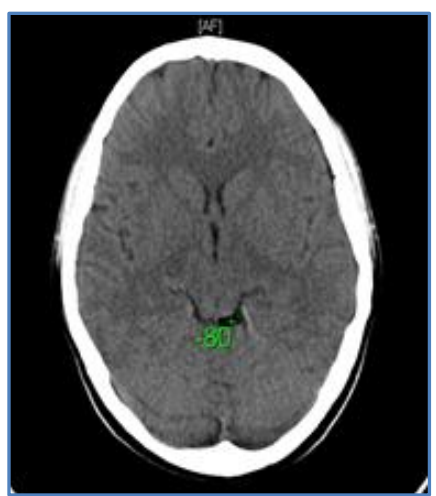

Fig. 2: CT scan showed fatdensity lesion (-80H.U) of the left quadrigeminal plate cistern

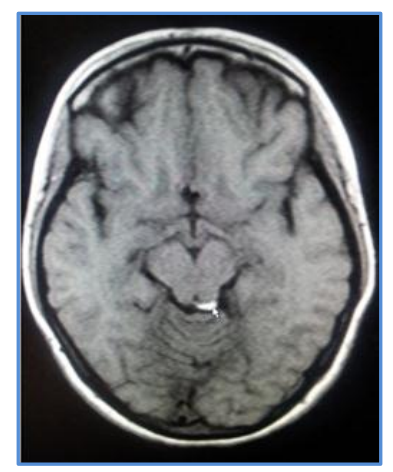

Fig. 3: Axial T1W image showing well defined hyperintense lesion in left quadrigeminal plate cistern (arrow)

\section{AUTHORS:}

1. Kiran Raju Nandyala

2. S. Venkateswar Rao

3. Shirish Paul Ganta

4. V. N. Narvekar

5. V. Sivaleela

\section{PARTICULARS OF CONTRIBUTORS:}

1. Assistant Professor, Department of Radiology, ASRAM Medical College.

2. Associate Professor, Department of Radiology, ASRAM Medical College.

3. Assistant Professor, Department of Radiology, ASRAM Medical College.

\section{FINANCIAL OR OTHER}

COMPETING INTERESTS: None
4. Professor, Department of Radiology, ASRAM Medical College.

5. Professor, Department of Radiology, ASRAM Medical College.

\section{NAME ADDRESS EMAIL ID OF THE CORRESPONDING AUTHOR:}

Dr. Kiran Raju Nandyala, \# B-11, Staff Quarters, ASRAM Medical College, Eluru-534005, West Godavari Distract, Andhra Pradesh. E-mail: rajkiren@gmail.com

Date of Submission: 06/02/2015. Date of Peer Review: 09/02/2015. Date of Acceptance: 10/03/2015. Date of Publishing: 23/03/2015. 